\title{
EDITORIAL
}

Check for updates

\section{Watching preprints evolve}

\author{
In February 2021, Nature Reviews Immunology launches the first of its monthly 'Preprint Watch' \\ columns. Here, we explain the rationale for our coverage of preprints and the precautions we \\ have taken to guard against their improper use.
}

Later this year, Nature Reviews Immunology will celebrate its 20th anniversary and it is fair to say that a lot has changed in our knowledge of the immune system since those early days. But what about the way in which immunology research is reported and published?

The first preprint server arXiv, which started life as an electronic bulletin board for physicists, launched in 1991, a decade before Nature Reviews Immunology. Similar preprint servers for biology and the life sciences (bioRxiv) and for the health sciences (medRxiv) were not launched until 2013 and 2019, respectively. Even then, uptake among the immunology community was slow; the number of bioRxiv preprints under the subject area 'immunology' increased from 2 in 2013 to 164 in 2017. However, the past few years have seen a rapid increase in preprint deposition among immunologists. The number of 'immunology' preprints deposited on bioRxiv was
521 in 2018, 929 in 2019 and 1,703 in 2020 (accessed on 14 December 2020). There has also been a proliferation of alternative preprint servers such as Research Square and Preprints.org. Since 2019, all Springer Nature journals, including Nature and its sister titles, have encouraged preprint submission of primary research manuscripts ${ }^{1}$.

There is no doubt that the COVID-19 pandemic, which necessitated the rapid dissemination of results, has fuelled some of this increase, with approximately one-quarter (463) of the 2020 'immunology' preprints on bioRxiv containing the search term 'COVID-19' or 'SARS-CoV-2'. It also seems clear from these numbers that even in non-pandemic times, preprints are here to stay. At Nature Reviews Immunology, we anticipate that the accelerated acceptance of the value of preprints that has occurred in 2020 will translate to long-term changes in their use, and we are well placed to respond to these changes.

\section{The value of preprints}

\section{Rapid and open}

the research community as well as the public got access to new research findings very rapidly

reflects the wish of society to get open and rapid access ... to public-funded research

a welcomed development, especially when fighting a public health crisis

\section{The future of science}

I think this approach will be utilized by many of us in the future

We are glad many of the major scientific journals became open to this concept

I post pretty much every research paper on preprint servers ... I also search through bioRxiv and medRxiv daily

\section{Networkins}

allow scientists, and particularly trainees, to share science without needing to await ... peer-review

They provide an instant source of information and democratize science dissemination

\section{Claim primacy}

an intermediate step to secure the so-called 'prior art' of a discovery

\section{Responsible approach}

I think most people have understood the difference between preprints and peer-reviewed papers

we take it as our responsibility to carefully contextualize and check what is shared in advance of formal review

\section{The need for caution}

\section{Information overload}

overflow of premature information

the descriptor-term 'avalanche' may actually be an understatement

\section{Premature, poor-quality information}

unprocessed information ... without proper interpretation

poor quality studies are being published

lack of 'quality control' of preprint releases

Fuels public mistrust

fire back in the face of science, with consequences for funding and general belief by the society

Non-scientists argue that they no longer can believe science

potentially feeding the community with wrong, incomplete and thus misleading information

may become abused by anti-science disinformation groups

Lack of peer review

numerous preprint publications, that created a lot of public attention ... which never made it through peer-review evaluation

the central role of peer-reviewed science through publication in good journals will remain very important

entails the great danger of 'relaxing' the rules of ethical research reporting under the peer-review system. 


4 we are
maintaining
a cautious
approach to pre-
prints, covering
only a few select
papers picked
by experts after
careful review

Recognizing the particular importance of preprints in the early stages of the COVID-19 pandemic, in April 2020 Nature Reviews Immunology began a collaboration with the Precision Immunology Institute at the Icahn School of Medicine, New York, USA (the Sinai Immunology Review Project; SIRP) ${ }^{2}$, to publish short 'In Brief' summaries (for example, REF. ${ }^{3}$ ) on a weekly basis of the most relevant, new COVID-19-related preprints (for example, REF. ${ }^{4}$ ), many of which have since been published in high-profile journals (for example, REF. $^{5}$ ). We were mindful of the potential dangers of highlighting and publicizing non-peer-reviewed results, but we were reassured by the system that SIRP had established to scan, filter and review preprints, involving both early career researchers (ECRs) and faculty members. This curation of the preprint literature at a time when journals and editors were overwhelmed with submissions was an essential service to the community, and the training in peer review for ECRs who were shut out of the lab should pay future dividends. In June 2020, a second group from the University of Oxford, UK (the OxImmuno Literature Initiative), operating under a similar system, also began to contribute regular preprint summaries (for example, $\mathrm{REFS}^{6,7}$ ).

These In Briefs have been well received online. We also know from speaking to our readers and authors that you see the value in rapid and open access to new results but that you are concerned by the volume and quality of some of these preprints. One of our authors described this as an 'overflow of premature information'. We share your concerns about media reporting of incomplete or misleading data that might damage public trust in science. For this reason, we are maintaining a cautious approach to preprints, covering only a few select papers picked by experts after careful review. We thank all those who have provided feedback to help us shape our coverage.

As the number, novelty and urgency of COVID-19related preprints decrease, it is time to announce the next phase in our preprint coverage, which is now extended to non-COVID-19 immunology preprints. Our aim is to continue providing our readers with a 'heads up' regarding early data, reliant on the expertise of our external collaborators but always caveated by the fact that these reports have not yet been through a 'traditional' peer review process. The teams from SIRP and OxImmuno have established a weekly virtual joint 'journal club' (the Oxford-Mount Sinai (OxMS) Preprint Journal Club), where they discuss selected preprints and, from these sessions, nominate two preprints per month to report on in Nature Reviews Immunology. These 'Preprint Watch' articles are a longer format than the In Briefs, which should allow for greater depth of discussion regarding the methods and results.

We cannot predict the future of preprints and we will, as always, be guided by the feedback that we receive, but we hope that you will find these useful and informative. We are also keen to make sure that this is a truly global community effort and we hope that, in the future, other groups will become involved. Through this system of independent evaluation and selection, we hope to play a small part in ensuring that the value of preprints outweighs their potential adverse effects.

1. Springer Nature journals unify their policy to encourage preprint sharing. Nature 569, 307 (2019).

2. Vabret, N. et al. Advancing scientific knowledge in times of pandemics. Nat. Rev. Immunol. 20, 338 (2020).

3. Catalan-Dibene, J. Human antibodies can neutralize SARS-CoV-2. Nat. Rev. Immunol. 20, 350 (2020).

4. Ju, B. et al. Potent human neutralizing antibodies elicited by SARS-CoV-2 infection. Preprint at bioRxiv https://doi.org/ 10.1101/2020.03.21.990770 (2020).

5. Ju, B. et al. Human neutralizing antibodies elicited by SARS-CoV-2 infection. Nature 584, 115-119 (2020).

6. Coveney, C. \& Alderson, J. SARS-CoV-2: too infectious to handle? Nat. Rev. Immunol. 20, 462 (2020).

7. University of Oxford Immunology network. SARS-CoV-2 RNA detected in blood samples from patients with COVID-19 is not associated with infectious virus. University of Oxford https:/ www.immunology.ox.ac.uk/covid-19/covid-19-immunologyliterature-reviews/sars-cov-2-rna-detected-in-blood-samplesfrom-patients-with-covid-19-is-not-associated-with-infectious-virus (2020). 